But these are still early-stage results. It is not clear yet whether the improvements are the result of the cells, or whether something else, such as the body's own regenerative capacity, was at work. To find out, the company wants permission to move forward with a randomized, controlled phase 2 clinical trial. That's the right way to do things: stepping carefully, slowly and rigorously forward.

Meanwhile, in Japan, a more worrisome approach is unfolding. Last month, researchers at Sapporo Medical University leapfrogged all other spinal-cord injury treatments that use stem cells - including the one being investigated by Asterias - and received market approval for injections of a type of cell called a mesenchymal stem cell. There are reasons to be sceptical, or at least to delay the sale of this procedure to patients.

The very nature of these cells - in particular, whether they function as stem cells and do turn into neurons as suggested by the Japanese group - is subject to fierce debate (D. Sipp et al. Nature 561, 455-457; 2018). The clinical trials that demonstrated efficacy were based on only 13 participants. There was no control group and the trial data remain unpublished.

Through a fast-track process for regenerative medicine, launched in 2014 to speed treatments to patients and spur innovation, the Japanese government gave the mesenchymal stem-cell treatment, called Stemirac, 'conditional approval' to enter the market. It can be sold to patients, and the company was given seven years to show that it works. How that evidence will be collected once the treatment is on the market is an unanswered question.

A better way would have been to run a randomized controlled clinical trial, with both participants and physicians unaware of who received the cells and who received a placebo. But under Japan's fasttrack system, researchers at the university didn't have to do this. The researchers also should have published the clinical data already collected, but in Japan they are discouraged from doing so.
This seems surprising. Some companies might not want to publish clinical results to protect their trade secrets. But in this case, it is Japan's health ministry that seems to be telling researchers not to publish data. Although it is not a blanket ban - the propriety of publishing such data is evaluated on a case-by-case basis - a ministry representative told Nature that in this instance it would be discouraged. That's because published data could be used as "promotional materials" and

"Japan could
and should
introduce a
better and more
transparent
system."
unduly influence patients or officials, according to the health ministry official.

Japan has set up a bizarre situation. The university has made promises about the treatment in an advertisement unencumbered by data, but the inclusion of scientific evidence, in a form that the world's experts can evaluate, is considered too potentially misleading to publish. The Kafkaesque logic at play here seems to be that promoting a medicine without data is better than promoting it with.

The Japanese team has promised results so dramatically convincing that controlled trials would be unnecessary. Let's hope that is the case. But it is more likely that ambiguous results from the uncontrolled trial will allow the treatment to continue in use indefinitely. That is fair neither to the patients who are willing to try the treatment, nor to other companies that are putting therapies through truly rigorous trials.

Japan could and should introduce a better and more transparent system, one that requires the production of sound clinical efficacy data through controlled trials, when possible, and that encourages broad evaluation of those data - in scientific publications, when feasible - by the international medical research community. Japan could, in other words, learn a lot from the way it's being done in California. Until then, offering people such stem-cell treatments is premature and unfair.

\section{Early detection}

\section{The growing capabilities of prenatal diagnostics are expanding the need for counselling.}

$\mathrm{T}$ here is perhaps no greater consumer of information than an expecting parent. Details abound about pregnancy, delivery, infant care and feeding, developmental milestones, and parenting philosophies. All are available to learn - and obsess over, for those so inclined.

That pool of information is now being topped up by a steady drip of genetic data. In coming years, as non-invasive methods of prenatal genetic testing become more sophisticated and expansive, that drip could become a flood. We need to prepare.

Perhaps the biggest priority should be the latest generation of techniques that sample snippets of fetal DNA found floating in a mother's blood. In some countries, the tests are increasingly being used to look for large chromosomal abnormalities, such as the extra copy of chromosome 21 that causes Down's syndrome. Such screening tools can reduce the use of more invasive tests, such as amniocentesis and chorionic villi sampling, which carry a small risk of miscarriage.

As the methods for isolating and sequencing such DNA improve, clinicians can gain information about more and more genetic disorders before birth. A study published this week (J. Zhang et al. Nature Med. http://doi.org/cz4m; 2019) demonstrates how far the technology has come - and where it is heading - by describing a test that can screen for 30 spontaneously arising single-gene disorders.

The group tested it on 422 samples from 131 clinics around the world. Some of the samples came from pregnancies with abnormal ultrasound findings, or a family history of disease. Others had no indication of trouble ahead. Thirty-two tested positive for a genetic disorder.
The team was able to follow up on outcomes from 233 of these pregnancies and found no false positive or false negative results among those for which diagnostic data were available. But the test would need to be repeated on a grander scale to nail down the risks that would come with positive findings, and to establish who would benefit most from the technology.

The expansion of non-invasive prenatal tests seems inevitable. This can benefit both parents and children. Prenatal testing could allow prospective parents and medical teams to prepare for the needs of the newborn, or, in some cases, to make the difficult decision to end a pregnancy. Many expectant parents welcome such tests. A survey of 186 women in the United States found that $83 \%$ thought prenatal tests that sequence all of the genome's protein-coding genes should be offered (E. J. Kalynchuk et al. Prenat. Diagn. 35, 1030-1036; 2015). Such thorough sequencing of circulating fetal DNA is too complex and expensive to be practical at present, but it will become easier and cheaper in the future.

Still, prenatal screening based on circulating fetal DNA rarely offers definitive diagnoses: it must often be followed up by other tests to confirm the result, so parents can end up worrying unnecessarily. Furthermore, the effects of some disease-associated genetic variants could be masked by other variants elsewhere in the genome, or by environmental factors. Both of these factors mean additional, sometimes invasive, tests are often needed to back up genetic findings - and in many cases, genetics alone will never offer a clear indicator of disease risk.

Researchers and clinicians must adapt to the changes that such tests will bring. Widespread use will increase demand for trained genetic counsellors, who are already in short supply. Efforts to educate physicians and patients will need to be expanded, and the costs of counselling will need to be included in price estimates for implementing the tests.

Meanwhile, clinicians, researchers, ethicists and the public need to come together to develop guidelines about when such testing should be deployed and for which conditions, and how the information should be handled once the results are in. 